\title{
Assessing satisfaction with desloratadine and fexofenadine in allergy patients who report dissatisfaction with loratadine Daniel J Glass* and Anne S Harper
}

\author{
Address: Zynx Life Sciences, Cerner Corporation, Beverly Hills, CA, USA \\ Email: Daniel J Glass* - dglass@cerner.com; Anne S Harper - aharper@cerner.com \\ * Corresponding author
}

This article is available from: http://www.biomedcentral.com/I47/-2296/4/10

(C) 2003 Glass and Harper; licensee BioMed Central Ltd. This is an Open Access article: verbatim copying and redistribution of this article are permitted in all media for any purpose, provided this notice is preserved along with the article's original URL.

\begin{abstract}
Background: The FDA recently moved loratadine (Claritin) from prescription only status to over-the-counter (OTC). In response to the availability of an OTC non-sedating antihistamine, many managed care organizations are reevaluating which if any prescription antihistamines should remain on formulary. From a managed care perspective, determining which of the remaining prescription antihistamines results in the greatest patient satisfaction with allergy treatment would be informative.
\end{abstract}

Methods: We report on a weighted cross sectional survey $(n=10,023)$ delivered online to a sample of allergy sufferers in the U.S. during the month of December 2002. Two segments were identified for analysis: patient who were dissatisfied with loratadine and converted to desloratadine (Clarinex; $n=6 I)$, and patients who were dissatisfied with loratadine and converted to fexofenadine (Allegra; $n=2 \mathrm{II}$ ). The two segments were compared along a series of measures that the literature suggests are related to treatment satisfaction.

Results: The survey found that two of the satisfaction measures differentiated desloratadine converters from fexofenadine converters $(p<.05)$ : mean sum of self-reported adverse events and nighttime awakening due to allergy symptoms. For the remainder of satisfaction measures though, patients who were dissatisfied with loratadine reported equal duration of coverage and satisfaction with desloratadine as fexofenadine. When severity of disease was controlled for in the analysis, a pattern emerged suggesting greater levels of satisfaction amongst loratadine dissatisfied patients who converted to desloratadine. Point estimates suggest a consistent pattern favoring desloratadine patient satisfaction, with statistically significant results reported for sum of adverse effects, nighttime awakening due to symptoms, symptom severity just prior to the next dose, and overall satisfaction $(p<0.05)$.

Conclusions: On average, patients who were dissatisfied with loratadine reported equal or better satisfaction with desloratadine as fexofenadine. Patients with severe allergic rhinitis reported greater satisfaction when converted from loratadine to desloratadine than fexofenadine for select satisfaction measures. These results suggest that if managed care intends to position prescription antihistamines as second line for OTC loratadine treatment dissatisfaction, desloratadine is a useful treatment alternative. These findings, while informative to formulary decision-makers, must be interpreted with caution. Only through head-to-head controlled clinical trials can differences in efficacy and safety be established. 


\section{Background}

The FDA recently allowed loratadine (Claritin) to move from prescription only status to over-the-counter (OTC). Given the high prevalence of allergic rhinitis in the population, this move has wide-ranging implications for both patients who are regular prescription allergy medication users as well as allergy sufferers who already rely on OTC products to treat their allergies. As of December, 2002 $15 \%$ of the U.S. prescription antihistamine market was allocated to loratadine use [1]. These patients are now faced with the option of OTC loratadine or a transition to another prescription antihistamine. For existing OTC users, a non-sedating antihistamine is now available without a doctor's prescription, thus increasing the convenience and availability of safe and effective allergy medications.

In response to the availability of an OTC non-sedating antihistamine, many managed care organizations are reevaluating which if any prescription antihistamines should remain on formulary. These decisions reflect, in part, a movement of brand-name antihistamines to tier three status on many formularies [2-4]. Prescription allergy medications such as fexofenadine (Allegra), desloratadine (Clarinex), and cetirizine (Zyrtec) (which represent over $75 \%$ of the U.S. prescription antihistamine market), [1] are increasingly being placed on the third tier of managed care drug benefit plans which requires the largest co-pay from patients. Patient co-payments at this benefit level can range from $\$ 30$ to $\$ 50$ dollars $[2,4]$, rendering prescription antihistamine treatment an expensive option for allergy sufferers. From a managed care perspective, shifting a greater proportion of the direct cost of allergy care to the patient can act as an incentive for patients to consider over-the-counter loratadine as first line therapy. Managed care's role in allergy treatment may therefore become more focused on providing treatment alternatives to those patients who do not find adequate symptom relief from loratadine. Therefore, determining which of the currently available prescription antihistamines results in the greatest patient satisfaction with allergy treatment (given dissatisfaction with loratadine treatment) would be informative as managed care formulary decision makers and benefit plan designers attempt to maximize enrollee health and satisfaction at the lowest cost.

To better understand patient satisfaction with available prescription antihistamines, this research examined satisfaction outcomes between patients who reported dissatisfaction with loratadine and switched to desloratadine, and patients who reported dissatisfaction with loratadine and switched to fexofenadine. Cetirizine (Zyrtec) was excluded from these analyses because its label reports an increased incidence of sedation. There is a body of litera- ture to suggest that untreated or under-treated allergic rhinitis is also associated with fatigue and sedation [14]. As a result, Zyrtec was excluded from the analysis because perceived efficacy is related to cognitive functioning and we assume that perceived efficacy is a dimension of patient satisfaction.

By focusing on patients who self-reported switching antihistamines due to dissatisfaction with loratadine treatment, the results of this study would be directly applicable to the patient population that managed care will potentially need to provide alternative allergy treatment options (e.g., prescription antihistamine, nasal steroids, immunotherapy). Additionally, we examine satisfaction outcomes between these patient segments for self-reported severe allergy sufferers, thus allowing us to explore the differential satisfaction outcomes within the patient population in most need of effective allergy treatment.

\section{Methods \\ Study Sample}

The results of this paper are drawn from a larger study, reflecting a nationally representative sample of U.S. allergy sufferers $(n=10,023)$ conducted online during the month of December, 2002. Data were weighted to reflect demographics of the U.S. adult population which suffers from seasonal or perennial allergies. Weighting targets were based on the results of the December, 2002 Harris Poll $^{\oplus}$, a telephone poll that randomly surveyed over 1,000 U.S. adults. All U.S. resident allergy sufferers over the age of 18 were included in the weighting. They were weighted by educational attainment, age by sex, race/ethnicity, region, and household income to be representative of the population of adult allergy sufferers in the U.S. This larger study design included over 18 patient segments, reflecting specific treatment practices, disease states, current medications, and medication switching behavior.

From this larger study, two patient segments were identified for analysis within this paper: patients who were dissatisfied with loratadine and converted to desloratadine (Clarinex; $\mathrm{n}=61$ ), and patients who were dissatisfied with loratadine and converted to fexofenadine (Allegra; $\mathrm{n}=$ 211). Qualification was determined in the screener section of the survey. In order to qualify for these segments, patients had to meet certain criteria. They had to have been on each medication (previous and current) for at least one week, with no more than one year elapsed between product usages, and the patient reported that the reason for switching products was dissatisfaction with loratadine. Patients who reported combination prescription product use did not qualify for these segments (e.g., prescription antihistamine use and prescription nasal steroid use). However, patients who reported concomitant OTC use were allowed in these segments. Once identified 
for qualification, patients completed survey questions which examined disease state and medication behavior, reasons for switching products, and satisfaction with previous and current medications.

\section{Study Measures}

The two segments were compared along a series of measures that the literature suggests are related to treatment satisfaction [5-7]. We asked patients to assess their product satisfaction across three categories: side effects associated with the medication, satisfaction with symptom relief, and overall satisfaction. For side effects, patients identified instances of adverse events or side effects associated with each medication experience. The list of side effects included the following: difficulty falling asleep, trouble sleeping through the night, trouble waking up, early wakening, daytime drowsiness, nervousness, mood swings, poor concentration, reduced ability to be productive at work/school, headache, nausea, fatigue, dry mouth, and other. For each respondent, we summed the recorded instances of medication-related side effects or adverse events and created a composite measure, reported as "sum of adverse events." All analyses examined mean differences in this measure between the two patient segments.

For satisfaction related to symptom relief, we asked respondents to assess their medication in five ways. Respondents were asked to indicate how effective their medication was in covering their symptoms through to the next dose, a measure we report as "coverage period." This measure is based on a seven point Likert scale, where 1 equals not effectively at all and 7 equals extremely effectively. Additionally, respondents were asked to indicate how quickly their medication begins to relieve their symptoms after they dose, a measure we report as "onset of relief." This measure is based on a seven point Likert scale, where 1 equals not quickly at all and 7 equals extremely quickly. Respondents were also asked to indicate how frequently their allergy symptoms prevented them from sleeping through the night, a measure we report as "end of dose failure - nighttime awakenings." This measure is based on a seven point Likert scale, where 1 equals not frequently at all and 7 equals extremely frequently. Respondents were further asked to indicate how troubled they were by their allergy symptoms when they get out of bed in the morning, a measure we report as "end of dose failure - morning symptoms." This measure is based on a seven point Likert scale, where 1 equals not troubled at all and 7 equals extremely troubled. And finally, respondents were asked to indicate their overall level of symptom relief they attained while taking their prescription medication, a measure we report as "overall symptom relief." This measure is based on a seven point Likert scale, where 1 equals no relief and 7 equals complete relief.
For overall prescription medication satisfaction, we asked respondents to indicate product satisfaction in four ways. Respondents were asked to indicate how likely they were to recommend their current prescription medication to a friend or family member, a measure we report as "likelihood to recommend." This measure is based on a seven point Likert scale, where 1 equals not likely at all and 7 equals extremely likely. Additionally, respondents were asked to indicate how likely they were to continue their current prescription medication, a measure we report as "continuation intentions." This measure is based on a seven point Likert scale, where 1 equals not likely at all and 7 equals extremely likely. Respondents were also asked to indicate their overall satisfaction with their current prescription medication, a measure we report as "overall satisfaction with current Rx." This measure is based on a seven point Likert scale, where 1 equals extremely unsatisfied and 7 equals extremely satisfied. And finally, respondents were asked to indicate their overall satisfaction with the change in prescription products, a measure we report as "satisfaction with Rx switch." This measure is based on a seven point Likert scale, where 1 equals extremely unsatisfied and 7 equals extremely satisfied. For a summary of the satisfaction Likert scales used in this analysis, see table 1.

The two patient segments were compared along the above listed satisfaction measures as complete segments and separately as severe sufferers within the two segments. Severity of disease was assessed on a self-reporting basis of how troubled the patient was by his or her allergies. Severe sufferers were identified by responses of quite a bit troubled, very troubled, or extremely troubled on a seven point Likert scale (top three box). For the severity subgroup analyses, the weighted segments sizes were 88 for loratadine-fexofenadine and 35 for loratadine-desloratadine.

To test for mean differences in the ten separate study measures, difference in means tests were conducted using the t-distribution. All significance testing was performed at the $95 \%$ confidence level, two-tailed.

Although respondents were asked to assess their prescription medication across categories related to perceived efficacy and side effects, an important caveat to highlight is the fact that patient perceptions regarding medication use captured through survey research cannot establish differences in the clinical properties related to efficacy or safety. The survey research reported here was designed to assess dimensions of satisfaction, across patient self-reported categories of side effects, efficacy, and overall product satisfaction. Only through head-to-head controlled clinical trials can differences in efficacy and safety be established. 
Table I:

\begin{tabular}{lcc}
\hline & Dimensions of Patient Satisfaction - Semantic Differential Likert Scales & Positive Endpoint 7 \\
\hline $\begin{array}{l}\text { Symptom Relief } \\
\text { EOD Failure - Nighttime Awakening }\end{array}$ & Negative Endpoint I & Extremely Frequently \\
EOD Failure - Morning Symptoms & Not Frequently at All & Extremely Troubled \\
Onset of Relief & Not Troubled at All & Extremely Quickly \\
Coverage Period & Not Quickly at All & Extremely Effectively \\
Overall Symptom Relief & Not Effectively at All & Complete Relief \\
Overall Satisfaction & No Relief & Extremely Likely \\
Continuation Intentions & & Extremely Likely \\
Likelihood to Recommend & Extremely Unlikely \\
Overall Satisfaction with Current Rx & Extremely Unlikely \\
Satisfaction with Rx Change & Extremely Unsatisfied & Extremely Satisfied \\
\hline
\end{tabular}

Table 2:

\begin{tabular}{|c|c|c|}
\hline & \multicolumn{2}{|c|}{ Loratadine Dissatisfied Segments } \\
\hline & $\begin{array}{l}\text { Converted to Fexofenadine } \mathrm{N}=21 \mathrm{I} \\
\text { (weighted) }\end{array}$ & Converted to Desloratadine $\mathrm{N}=6 \mathrm{I}$ (weighted) \\
\hline \multicolumn{3}{|l|}{ Side Effects } \\
\hline Sum of Adverse Events 1 & $1.59 *$ & $1.32 *$ \\
\hline \multicolumn{3}{|l|}{ Symptom Relief } \\
\hline EOD Failure - Nighttime Awakening' & $2.26 *$ & $1.85^{*}$ \\
\hline EOD Failure - Morning Symptoms ${ }^{1}$ & 3.33 & 3.12 \\
\hline Onset of Relief ${ }^{2}$ & 4.56 & 4.72 \\
\hline Coverage Period ${ }^{2}$ & 4.84 & 5.02 \\
\hline Overall Symptom Relief ${ }^{2}$ & 5.30 & 5.35 \\
\hline \multicolumn{3}{|l|}{ Overall Satisfaction } \\
\hline Continuation Intentions ${ }^{2}$ & 5.39 & 5.44 \\
\hline Likelihood to Recommend ${ }^{2}$ & 5.06 & 5.38 \\
\hline Overall Satisfaction with Current $\mathrm{Rx}^{2}$ & 5.13 & 5.28 \\
\hline Satisfaction with Rx Change ${ }^{2}$ & 5.17 & 5.32 \\
\hline
\end{tabular}

$*_{\mathrm{p}}<=.05{ }^{*} \mathrm{p}<=.01$ ' Higher number reflects negative medication experience. ${ }^{2}$ Higher number reflects positive medication experience.

Table 3:

$$
\text { Loratadine Dissatisfied Segments - Severe Allergic Rhinitis }
$$

Converted to Fexofenadine $\mathrm{N}=88$ (weighted) Converted to Desloratadine $\mathrm{N}=35$ (weighted)

\begin{tabular}{|c|c|c|}
\hline \multicolumn{3}{|l|}{ Side Effects } \\
\hline Sum of Adverse Events 1 & $2.0^{* * *}$ & $1.35^{* *}$ \\
\hline \multicolumn{3}{|l|}{ Symptom Relief } \\
\hline EOD Failure - Nighttime Awakening & $2.93 * *$ & $1.81 * *$ \\
\hline EOD Failure - Morning Symptoms ${ }^{\circ}$ & 3.74 & 3.62 \\
\hline Onset of Relief 2 & 4.34 & 4.58 \\
\hline Coverage Period ${ }^{2}$ & $4.37^{* *}$ & $5.15^{* *}$ \\
\hline Overall Symptom Relief ${ }^{2}$ & 4.92 & 5.34 \\
\hline \multicolumn{3}{|l|}{ Overall Satisfaction } \\
\hline Continuation Intentions ${ }^{2}$ & 5.10 & 5.41 \\
\hline Likelihood to Recommend ${ }^{2}$ & 4.66 & 5.32 \\
\hline Overall Satisfaction with Current $R x^{2}$ & $4.67^{*}$ & $5.37 *$ \\
\hline Satisfaction with Rx Change ${ }^{2}$ & 4.76 & 5.27 \\
\hline
\end{tabular}

$*_{\mathrm{p}}<=.05{ }^{*} \mathrm{p}<=.01{ }^{\prime}$ Higher number reflects negative medication experience. ${ }^{2}$ Higher number reflects positive medication experience. 


\section{Results \\ Full Segments}

When examining the full segments, the survey found that two of the satisfaction measures differentiated desloratadine converters from fexofenadine converters $(\mathrm{p}<.05)$ : mean sum of adverse events and end of dose failure nighttime awakenings. Patients who switched to desloratadine experienced significantly less instances of medication-related adverse events, when compared with patients who switched to fexofenadine (see table 2). Additionally, loratadine-desloratadine patients reported significantly less frequent nighttime awakenings due to allergy symptoms (see table 2). As this measure gauges the medication's coverage period of symptom relief, this differential outcome speaks to the greater satisfaction associated with the buffering of end of dose failure symptoms for desloratadine when compared with fexofenadine.

For the remainder of the satisfaction measures, patients who were dissatisfied with loratadine and switched to desloratadine reported equal outcomes when compared with patients who switched to fexofenadine. However, as table 2 shows, point estimates for these satisfaction measures reveal a consistent pattern favoring desloratadine outcomes, although differences were not found to be statistically significant. In short, for these measures, the data clearly suggest that fexofenadine does not offer a better satisfaction outcome for patients who are dissatisfied with loratadine, when compared with desloratadine.

\section{Severe Allergy Sufferer Segments}

When severe allergy sufferers were analyzed separately, a pattern emerged suggesting greater levels of satisfaction amongst loratadine dissatisfied patients who converted to desloratadine when compared with loratadine dissatisfied patients who converted to fexofenadine. Point estimates again suggest a consistent pattern favoring desloratadine patient satisfaction, with statistically significant results reported for sum of adverse events, end of dose failure nighttime awakenings, end of dose failure - morning symptoms, and overall satisfaction with current $\mathrm{Rx}(\mathrm{p}<$ 0.05). As with the full segments, severe allergy sufferers who switched from loratadine to desloratadine due to dissatisfaction reported significantly less instances of medication-related adverse events, when compared with similar patients who switched to fexofenadine (see table 3 ). For the two end of dose failure satisfaction measures, loratadine-desloratadine patients reported significantly less frequent nighttime awakenings due to allergy symptoms as well as being significantly less troubled by their allergy symptoms upon morning awakening (see table 3 ). For overall satisfaction, severe loratadine-desloratadine patients reported significantly greater levels of satisfaction with their current antihistamine when compared with severe loratadine patients who switched to fexofenadine.
In total, when the satisfaction-related categories of reported medication side effects, assessments of symptom relief, and reports of overall satisfaction were considered, severe patients who had switched to desloratadine reported increased satisfaction when compared with severe patients who switched to fexofenadine.

\section{Discussion}

The results of this study show that for patients who report dissatisfaction with loratadine, a product switch to desloratadine offers equal, and in many instances, increased benefits along a series of product-related satisfaction measures when compared to a switch to fexofenadine. When all patients were considered, the most striking finding involved the significantly reduced frequency of nighttime awakenings due to allergy symptoms for desloratadine outcomes. As previous research has documented the importance of sleeping through the night for allergy sufferers [8], this finding speaks to a crucial component of product satisfaction favoring desloratadine conversion. Indeed, when severe sufferers were examined separately, both satisfaction measures related to end of dose failure favored desloratadine outcomes. Severe sufferers are arguably more likely to be awakened in the middle of the night due their severe symptoms as well as being more likely to report severe symptoms upon awakening [9-14]. As a result, an antihistamine which correlates with greater satisfaction along these coverage period dimensions would be valued by the severe allergy sufferer. To that end, the results of this survey suggest that desloratadine offers that benefit, when compared with fexofenadine (for severe allergy sufferers who failed on loratadine). This finding supports other research that highlights the 24 hour coverage associated with desloratadine $[15,16])$

The survey findings also reveal that severe sufferers who were dissatisfied with loratadine and switch to desloratadine report greater levels of satisfaction when compared with patients who switch to fexofenadine. This global assessment of satisfaction speaks favorably to the overall outcomes of desloratadine conversion, when specifically targeting patients who suffer from severe allergy symptoms. As severe allergy sufferers have the most to gain from allergy medication, this finding positions desloratadine as a beneficial treatment approach for severe sufferers who gain little satisfaction from loratadine use.

\section{Limitations}

One limitation of this study is that it only focuses on two product switching pathways. Although loratadine, fexofenadine, and desloratadine represented close to twothirds of the U.S. antihistamine market at the time of the survey fielding, knowledge about patients who are dissatisfied with loratadine and switch to cetirizine is unknown 
at this time. Understanding this specific switching pathway represents fruitful grounds for future research. Another study limitation involves the interpretation of data associated with product switchers. It must be acknowledged that inferences from these data can only be applied to a specific sub-segment of the antihistamine market, namely those allergy patients who were dissatisfied with loratadine and converted to either fexofenadine or desloratadine. Interpretations beyond this population would be suggestive in nature, and should be approached with caution.

\section{Conclusions}

On average, patients who were dissatisfied with loratadine reported equal or better satisfaction with desloratadine as fexofenadine. Patients with severe allergic rhinitis reported greater satisfaction when converted from loratadine to desloratadine than fexofenadine for select satisfaction measures. These results suggest that if managed care intends to position prescription antihistamines as second line for patients who are not satisfied with OTC loratadine, desloratadine is a useful treatment alternative. These findings, while informative to formulary decision-makers, must be interpreted with caution. Only through head-tohead controlled clinical trials can differences in efficacy and safety be established.

\section{Competing Interests}

None declared.

\section{Authors' Contributions}

DG performed all data analyses and drafted the manuscript. AH conceived of the study, and was responsible for its design and coordination. All authors read and approved the final manuscript.

\section{Acknowledgements}

This study was funded by the Schering Plough Corporation and completed by the Harris Interactive Health Care Division.

\section{References}

I. IMS Health. U.S. NRX Data 2002.

2. Segedin DA: Three-tied copayment plans: design considerations and effectiveness. Drug Benefit Trends 1999, I I (9):43-52.

3. Segedin DA: Health systems/plans share common coverage vision for Clarinex, OTC Claritin. Formulary 2002, 37(5):229.

4. Nair Kavita V, Ganther Julie M, Valuck Robert J, Mccollum Marrianne $M$ and Lewis Sonya J: Improving formulary compliance with managed care members. Drug Benefit Trends 2002, I4(7):44-46.

5. Juniper EF, Willms DG, Guyatt GH and Ferrie PJ: Aqueous beclomethasone dipropionate nasal spray in the treatment of seasonal (ragweed) rhinitis. Journal of the Canadian Medical Association 1992, I 47(6):887-892.

6. Ricard N, Kind P, Christian S, Jensen M and Stewart J: Link between patient preferences and treatment outcomes in seasonal allergic rhinitis: an empirical investigation. Clinical Therapeutics 1999, 21 (I):

7. Weaver M, Patrick DL, Markson LE, Martin D, Frederic I and Berger $M$ : Issues in the measurement of satisfaction with treatment. American Journal of Managed Care 1997, 3(4):579-94.
8. Craig TJ, Mende C, Hughes K, Kakumanu S, Lehman EB and Chinchilli $\checkmark$ : The effect of topical nasal Fluticasone on objective sleep testing and the symptoms of rhinitis, sleep, and daytime somnolence in perennial allergic rhinitis. Allergy Asthma Proc 2003, 24(I):53-8.

9. Craig TJ, Teets S, Lehman EB, Chinchilli VM and Zwillich C: Nasal congestion secondary to allergic rhinitis as a cause of sleep disturbance and daytime fatigue and the response to topical nasal corticosteroids. J Allergy Clin Immunol 1998, I 1 (5):633-637.

10. Lavie P, Gertner R, Zomer J and Podoshin L: Breathing disorders in sleep associated with "microarousals" in patients with allergic rhinitis. Acta Otolaryngol I 98I, 92(5-6):529-533.

II. Kushida CA, Guilleminault C, Clerk AA and Dement WC: Nasal obstruction and obstructive sleep apnea: a review. Allergy Asthma Proc 1997, 18(2):69-7I.

12. Young $T$, Finn $L$ and Kim $\mathrm{H}$ : Nasal obstruction as a risk factor for sleep-disordered breathing. The University of Wisconsin Sleep and Respiratory Research Group. J Allergy Clin Immunol 1997, 99(2):S757-762.

13. Reinberg A, Gervais $P$ and Levi F: Circadian: and cirannual rhythms of allergic rhinitis: an epidemiological study involving chronobiologic methods. J Allergy Clin Immunol 1 988, 81:57-62.

14. Spaeth J, Klimek I and Mosges R: Sedation in allergic rhinitis is caused by the condition and not by antihistamine treatment. Allergy 1996, 51:893-906.

15. Sabbah A: The place of new antihistamines in allergy management: apropos of Desloratadine. Allerg Immunol 2002, 34(10):377-83.

16. Meltzer EO, Prenner BM, Nayak A and and the Desloratadine Study Group: Efficacy and tolerability of once-daily $5 \mathrm{mg}$ desloratadine, an $\mathrm{HI}$-receptor antagonist, in patients with seasonal allergic rhinitis. Clin Drug Invest 200I, 2I(I):25-32.

\section{Pre-publication history}

The pre-publication history for this paper can be accessed here:

http://www.biomedcentral.com/1471-2296/4/10/prepub 